\title{
Autoimmune response to transthyretin in juvenile idiopathic arthritis
}

\author{
Cristina C. Clement, ${ }^{1}$ Halima Moncrieffe, ${ }^{2}$ Aditi Lele, ${ }^{2}$ Ginger Janow, ${ }^{3}$ Aniuska Becerra, ${ }^{4}$ \\ Francesco Bauli, ${ }^{1}$ Fawzy A. Saad, ${ }^{1}$ Giorgio Perino, ${ }^{5}$ Cristina Montagna, ${ }^{6}$ Neil Cobelli, ${ }^{7}$ John Hardin, ${ }^{7}$ \\ Lawrence J. Stern, ${ }^{4}$ Norman Ilowite, ${ }^{3}$ Steven A. Porcelli, ${ }^{8}$ and Laura Santambrogio ${ }^{1,7,8}$ \\ 'Department of Pathology, Albert Einstein College of Medicine, New York, New York, USA. ${ }^{2}$ Center for Autoimmune \\ Genomics and Etiology, Cincinnati Children's Hospital Medical Center, Cincinnati, Ohio, USA. ${ }^{3}$ Department of Pediatric \\ Rheumatology, Montefiore Medical Center, New York, New York, USA. ${ }^{4}$ Department of Pathology, University of \\ Massachusetts Medical School, Worcester, Massachusetts, USA. ${ }^{5}$ Department of Pathology, Hospital for Special Surgery, \\ New York, New York, USA. 'Department of Genetics, Albert Einstein College of Medicine, New York, New York, USA. \\ 'Department of Orthopedic Surgery, Montefiore Medical Center, New York, New York, USA. ${ }^{8}$ Department of Microbiology \\ and Immunology, Albert Einstein College of Medicine, New York, New York, USA.
}

Juvenile idiopathic arthritis (JIA) is the most common pediatric rheumatological condition. Although it has been proposed that JIA has an autoimmune component, the autoantigens are still unknown. Using biochemical and proteomic approaches, we identified the molecular chaperone transthyretin (TTR) as an antigenic target for B and T cell immune responses. TTR was eluted from IgC complexes and affinity purified from 3 JIA patients, and a statistically significant increase in TTR autoantibodies was observed in a group of 43 JIA patients. Three cryptic, HLA-DR1-restricted TTR peptides, which induced CD4 ${ }^{+} \mathrm{T}$ cell expansion and IFN- $\gamma$ and TNF- $\alpha$ production in 3 out of 17 analyzed patients, were also identified. Misfolding, aggregation and oxidation of TTR, as observed in the synovial fluid of all JIA patients, enhanced its immunogenicity in HLA-DR1 transgenic mice. Our data point to TTR as an autoantigen potentially involved in the pathogenesis of JIA and to oxidation and aggregation as a mechanism facilitating TTR autoimmunity.

Conflict of interest: The authors have declared that no conflict of interest exists.

Submitted: November 19, 2015 Accepted: January 29, 2016 Published: February 25, 2016

Reference information: JCI Insight. 2016;1(2):e85633. doi:10.1172/jci.insight.85633.

\section{Introduction}

Juvenile idiopathic arthritis (JIA) affects approximately 300,000 children in the United States (1-12). JIA is a heterogeneous disease; oligoarticular and polyarticular subtypes are the most common, and the systemic subtype has extra-articular features, including fever and rashes $(3,9,11)$.

Some evidence supports the notion that JIA is an antigen-driven, lymphocyte-mediated autoimmune disease (13), including the known association with certain HLA haplotypes and the high numbers of infiltrating T cells within arthritic joints (13-18). Currently, the putative autoantigens driving the T cell-mediated immune response are unknown.

In addition to $\mathrm{T}$ cell infiltration, there is significant evidence implicating $\mathrm{B}$ cells and autoantibodies in JIA pathogenesis; B cell depletion therapy is an effective treatment for both JIA and its associated uveitis, and the lesions of anterior uveitis show prominent B cell infiltrates and immunoglobulin deposition (19, 20). Additionally, transcriptional analysis of PBMCs from patients with oligo-JIA has shown increased markers for B cell activation (21).

Recently, reports identified transthyretin (TTR) as one of the proteins upregulated in the synovial fluid (SF) of patients with rheumatoid arthritis (RA) and osteoarthritis (OA) and as a possible target of their autoantibody response (22-25). It was also reported that amyloid deposits, which included TTR, were present on the synovial membrane (22) and that TTR is a potential biomarker in JIA-associated uveitis (26). However, a potential adaptive immune response to TTR and the molecular mechanism(s) involved in a TTR immune response are still unknown.

In the current study, we have used a combination of biochemical and proteomic approaches to characterize the adaptive immune response to TTR in patients with JIA. We detected a statistically significant increase in TTR production and in TTR autoantibodies in the JIA population as compared with controls. Additionally, we identified 3 naturally processed cryptic TTR peptides that bind to HLA-DR1 with high affinity and induce $\mathrm{T}$ cell proliferation and cytokine production in a small subset of JIA patients. Aggre- 
gated and oxidized TTR was observed in the SF of all analyzed JIA patients as compared to controls. The increased immunogenicity of carbonylated and misfolded TTR, compared to the native protein, was confirmed by immunization of HLA-DR1 transgenic mice. Our findings provide evidence of a role for TTR as an autoantigen potentially involved in the pathogenesis of JIA and suggest a role for protein oxidation or other posttranslational modifications (PTMs) in stimulating autoimmune responses targeting this protein.

\section{Results}

Global proteomic profiling of SF from JIA. As a first step toward the characterization of self antigens that could play a role in JIA pathogenesis, we performed a global proteomic profiling of the SF (Supplemental Tables 1 and 2; supplemental material available online with this article; doi:10.1172/jci.insight.85633DS1). Fifty micrograms of SF proteins, albumin and IgG precleared, were fractionated by $4 \%$ to $20 \%$ SDS-PAGE, followed by in-gel trypsin digestion and nanospray-liquid chromatography-linear trap quadrupole-tandem mass spectrometry (nanoLC LTQ MS/MS) sequencing. A total of 391 proteins were identified in the JIA SF from all 7 patients at more than 95\% probability (Supplemental Table 2, A-D, and Supplemental Figure 1A).

The GO annotations pinpointed many proteins that were significantly associated with acute-phase response, complement activation, coagulation, and immunoglobulin production (Supplemental Table 2, A-D, and Supplemental Figure 1B). Protein clusters associated with oxidative stress, macrophage and dendritic cell activation, and IL-12 and IL-17 production were also highlighted as further indications of active joint inflammation (Supplemental Table 2, A-D, and Supplemental Figure 1B). As expected, the proteomic analyses of JIA SF also showed the presence of several metalloproteases and other proteases, including plasmin kallikrein and cathepsins, in addition to tissue inhibitors of metalloproteinases (Supplemental Table 2, A-D). Altogether, the proteomic data confirmed the presence of an ongoing inflammatory process and overlapped substantially with previously reported proteomic analyses performed on SF of patients with RA and JIA $(4,11,27)$.

Peptidomic profiling of SF from JIA. Since the JIA-associated inflammation is characterized by extensive synovial and cartilage damage, we also performed a peptidomic analysis to map the fragments of degraded cartilage proteins (Figure 1; Supplemental Figures 2 and 3; Supplemental Tables 3 and 4; and refs. 8, 28-30). A total of 788 unique naturally processed endogenous peptides derived from 108 proteins were identified by the combined analysis of SF from 7 JIA patients (Figure 1A; Supplemental Table 3, A and C; and Supplemental Table 4, B and C). In contrast, we found only 71 unique peptides derived from the processing of 23 proteins in the combined analysis of the control samples (Figure 1A; Supplemental Table 3, B and D; and Supplemental Table 4, A and C).

All protein substrates that generated the SF peptidome of JIA and control subjects were used to generate the hierarchical cluster analysis (Figure 1B and Supplemental Table 3F). The peptide substrates with the most statistically significant contributions to the peptidome of JIA versus control subjects are depicted as a cluster (at $0.0<P<0.08$ ) and reveal disease-associated potential biomarkers (Figure 1B). These include apolipoproteins (AI and C-I), fibrinogen $\beta$ chain, fibronectin, kininogen-1, collagen $\alpha-1$ (III), fibrinogen $\gamma$ chain, $\alpha-1$ antitrypsin, TTR, and complement C4-B. Some of these proteins are already known to be putative or validated biomarkers of rheumatic diseases $(4-6,8,10,11,31)$.

The majority of JIA-associated peptides were derived from the processing of the acute-phase response proteome as well as from cartilage degradation (Figure 1, C and D; Supplemental Table 3, A and C; and Supplemental Figures 2 and 3). Indeed, peptide fragments of extracellular matrix proteins, including laminin subunit $\gamma-3$, syndecan-3, fibronectin, and mucin-16, and many subclasses of collagens (types I, II, III, IV, V, IX, X, XV, XVIII) were mapped (Figure 1; Supplemental Table 3, A, C, and E; and Supplemental Table 4, B and $\mathrm{C}$ ). On the other hand, proteins found in the control peptidome mostly derived from serum components (haptoglobin, hemoglobin albumin) or from proteins released by tissue damage (histones), as expected in SF collected following trauma (Figure 1; Supplemental Table 3, B and D; and Supplemental Table 4, A and C).

Enzymes generating the SF peptidome/degradome. To gain further understanding of the putative processing pathways and enzymes involved in the generation of the endogenous processed peptides found in the JIA SF, we screened the MEROPS and CutD databases, which report peptide cleavage sites that were previously experimentally determined and confirmed by N-terminal sequencing, tandem mass spectrometry (MS/ MS), or SILAC-based experiments $(32,33)$. This analysis identified 64 peptides with cleavage sites that had been previously mapped and indicated that a wide variety of proteases are involved in the generation of the JIA SF peptidome. Among these, matrix metalloproteases and cathepsins are highly represented, 
A

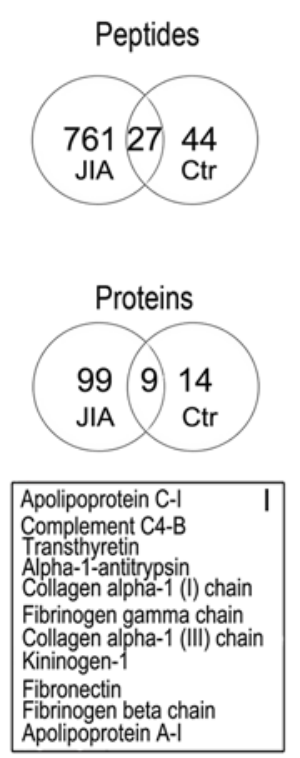

B

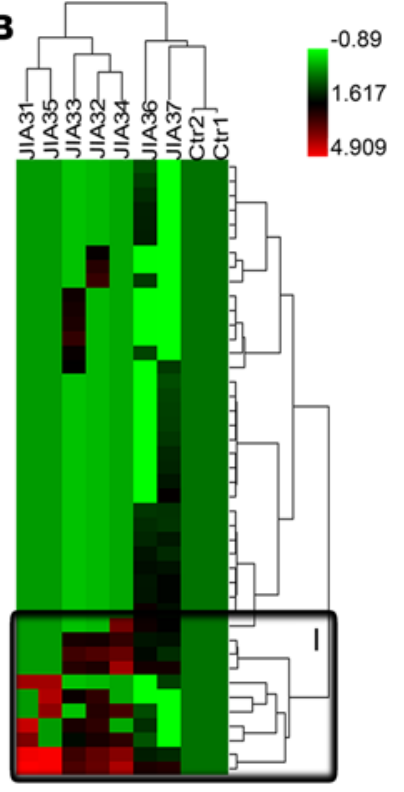

D

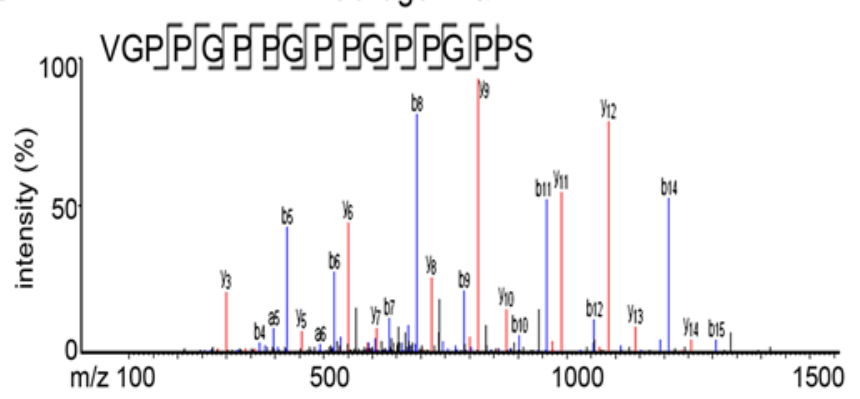

Transthyretin

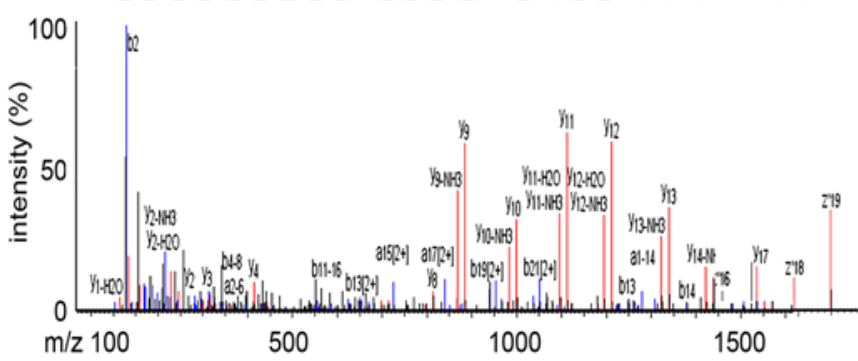

C Apolipoprotein CIII! Acute Phase Response

Apolipoprotein $\mathrm{Cl}=\mathrm{Ctr}$

Apolipoprotein B100 I

Apolipoprotein A-IV I

Apolipoprotein A-II I

Apolipoprotein A-I

Transthyretin

Serum amyloid A4

Albumin

Serotransferrin

Kininogen-1

a2HS glycoprotein

a 2 macroglobulin

Haptoglobin-related

Haptoglobin

\# unique peptides: $0 \quad 20 \quad 40 \quad 60$

Syndecan $3 \square \quad$ Matrix proteins

Laminin $\gamma^{3}$ ।

Mucin 16

Fibronectin

Collagen IX $\alpha 3$ ।

Collagen IV $\alpha 6$ ।

Collagen IV $\alpha 5$

Collagen IV $\alpha 1$ |

Collagen $V \alpha .1$ ॥

Collagen $\mathrm{V} \alpha 2$ ।

Collagen III $\alpha 1$

Collagen II $\alpha 1$

Collagen $\mid \alpha 2$

Collagen I $\alpha 1$

\# unique peptides: 0

\section{IA}


A

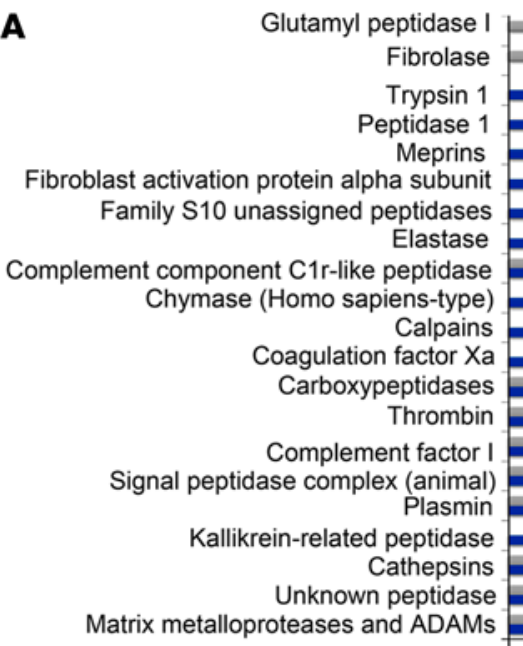

= Control

- JIA

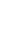




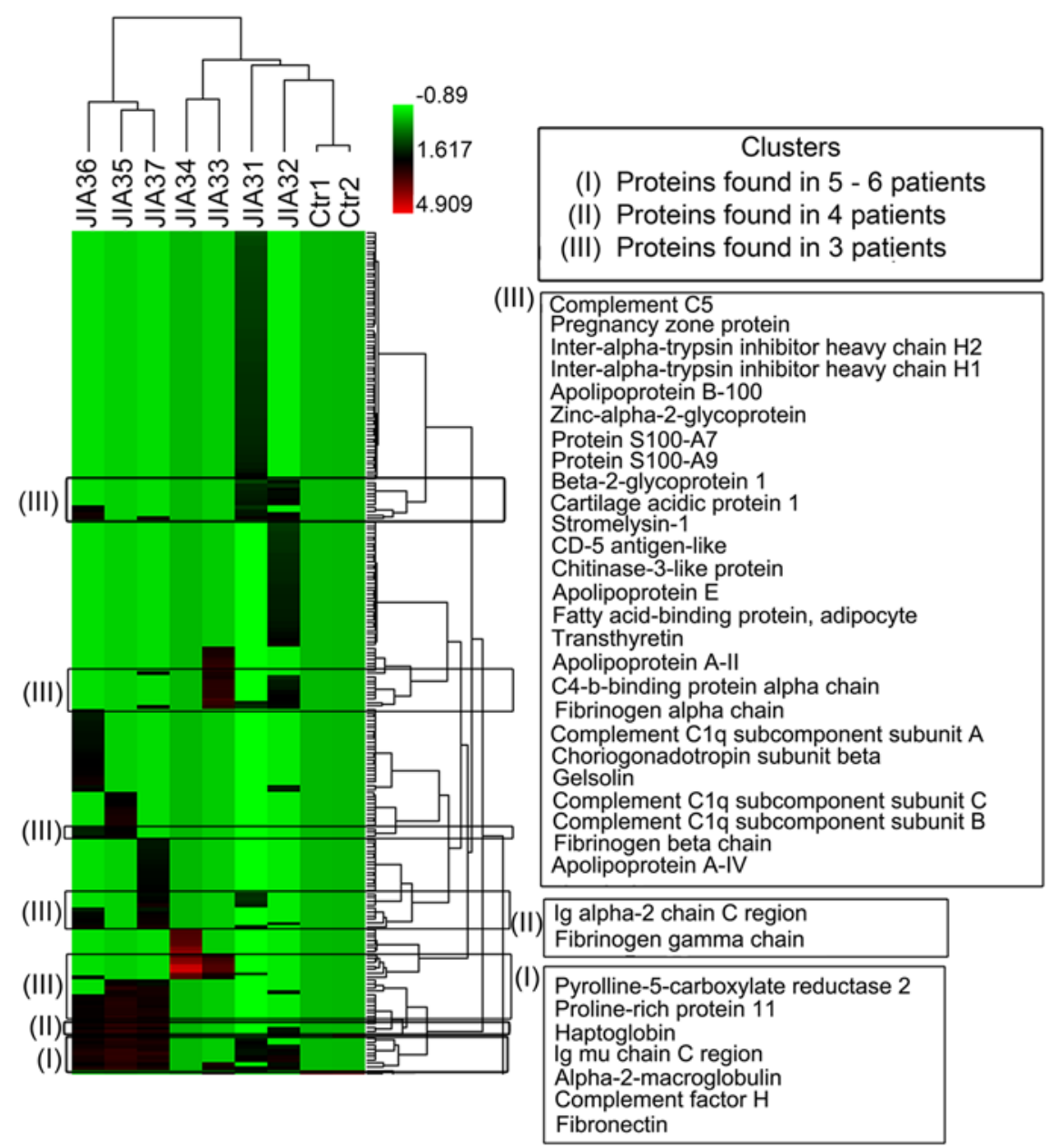

Figure 3. IgG-eluted immune proteome from the synovial fluid of patients with juvenile idiopathic arthritis and controls. Hierarchical cluster analysis (HCA) of proteins eluted from the IgG purified from the synovial fluid of patients with juvenile idiopathic arthritis (JIA) and controls (data were compiled from 3 separate proteomic analyses). Quantitative data sets were generated for each protein entry in Scaffold 4 using the log of the normalized spectral abundance factor [In(NSAF)] values [average of 3 experiments for the In(NSAF)]. The Ward's dual-clustering method followed by the $t$ test was performed for comparison of 2 controls and 7 JIA patients. The HCA depicts proteins with at least a 1.5 -fold difference in expression between the JIA and the control groups, with statistical significance corresponding to a FDR value $<0.7$ [green corresponds to the lowest In(NSAF), while red corresponds to the highest In(NSAF)] (proteomic data used to compile the heat map are reported in Supplemental Table 5).

As calculated, Pro oxidations encompassed over $16 \%$ of the total JIA peptidome, followed by Met oxidations (about 10\%) and combined Lys and Arg oxidations (about 3.5\%). As compared with the endogenous processed peptides found in the SF of OA and RA patients (37), the citrullination of Arg represented around $2 \%$ of the total JIA peptidome (Figure $2 \mathrm{~B}$ ). The carbonylation of Arg (Arg conversion into the glutamic semialdehyde) represented $1 \%$, while the combined Trp conversion into oxolactone and kynurenine was about $0.4 \%$ in the total JIA peptidome. Overall the citrullination of Arg was absent in the control patients, and all carbonylation and oxidations were significantly lower in the peptidomes of controls (Figure 2B and Supplemental Table 3A).

We further validated the MS/MS-mapped increased carbonyl content in the JIA SF peptidome as compared with the control SF peptidome using a spectroscopic method that detects carbonylated proteins. Equal amounts of total protein from the SF of JIA patients and controls (10-20 micrograms) were derivatized with dinitrophenylhydrazine (DNPH), and the total carbonyl content (as nanomoles/mg protein) was determined spectroscopically. A significantly higher amount of carbonylated proteins was detected in the JIA SF compared with that in the control SF (Figure 2C). Altogether, our analysis highlights the extent of the oxidative modifications associated with the self peptidome mapped in the SF of JIA patients during inflammation.

Profiling of immune complex-associated antigens in JIA SF. To focus on self antigens targeted by the autoimmune response in JIA, we characterized the proteins bound to immune complexes that are abundant in 
A

Synovial fluid (anti-TTR Ab)

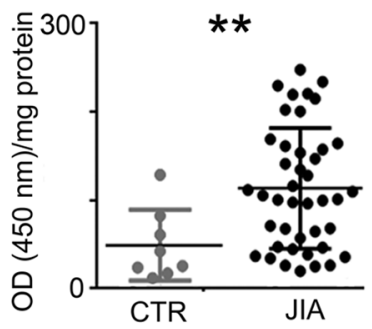

B

Sera (anti-TTR Ab)

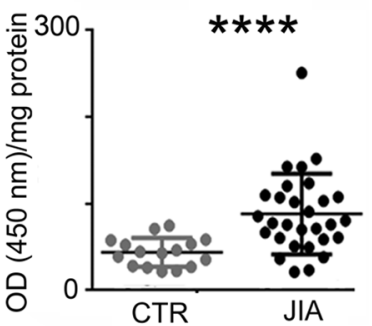

C

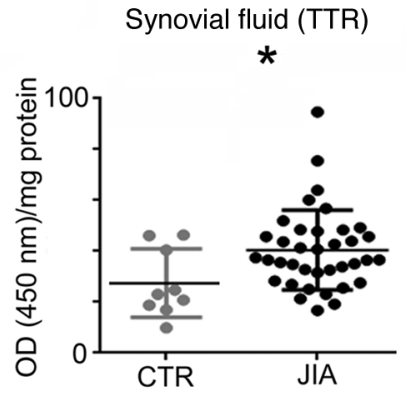

D

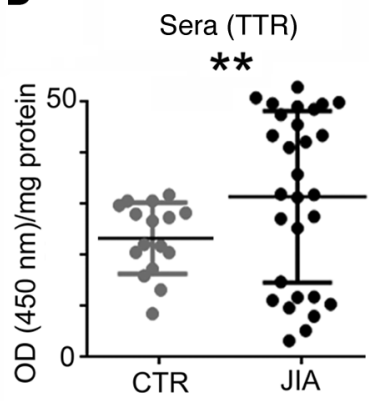

$\mathbf{E}$

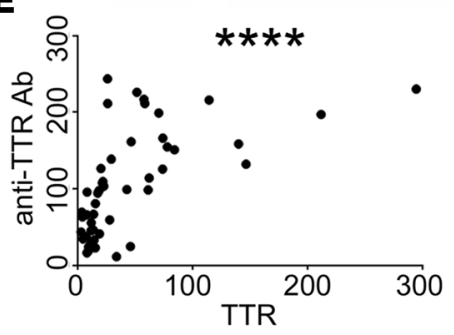

Figure 4. Transthyretin and anti-transthyretin antibodies in the synovial fluid and sera of juvenile idiopathic arthritis patients and controls. (A) Quantification of transthyretin (TTR) autoantibodies, as detected by ELISA, present in the synovial fluid from patients with juvenile idiopathic arthritis (JIA) and controls (data were compiled from 3 separate ELISAs, with each patient's samples run in quadruplicate). Data were analyzed by 1-way ANOVA and Tukey test. (B) Quantification of TTR autoantibodies, as detected by ELISA, present in the sera of patients with JIA and controls (data were compiled from 3 separate ELISAs, with each patient's samples run in quadruplicate). Data were analyzed by 1-way ANOVA and Tukey test. (C and D) Amount of TTR present in the synovial fluid and sera from patients with JIA and controls (data were compiled from 3 separate ELISAs, with each patient's samples run in quadruplicate) Data were analyzed by 1-way ANOVA and Tukey test. (E) Correlation between TTR protein and anti-TTR antibodies (Spearman $r=0.72$, $\left.{ }^{* * * *} P<0.001\right)$. The patient ID for each circle is reported in Supplemental Figure 4. Mean \pm SD. ${ }^{*} P<0.05 ;{ }^{* *} P<0.005 ;{ }^{* * *} P<0.001$.

the SF of JIA patients (Figure 3). Immunoglobulins in the SF of controls and JIA patients were purified using protein $\mathrm{A} / \mathrm{G}$ gel beads, and self antigens were eluted at low $\mathrm{pH}$ and analyzed by nanoLC LTQ MS/ MS sequencing. Using this approach, we identified 32 proteins in the JIA group that were absent from the control group and thus were putative disease-associated markers (Figure 3 and Supplemental Table 6).

The heat map analysis presented in Figure 3 highlights these antibody-targeted antigens, which included fibronectin, $\beta$-2 glycoprotein 1 , and proteins S100, A7/9, and TTR, among others.

TTR as a target antigen in JIA. Previous reports identified TTR as a target of an autoantibody response in OA and RA and as intraocular biomarker in JIA-associated uveitis (23-27). Indeed, we mapped TTR in the SF proteome/peptidome (Figure 1, Supplemental Table 2, and Supplemental Figure 1) and as one of the IgG-bound antigens (Figure 3 and Supplemental Table 6). To validate TTR as a target of the antibody response, we performed ELISA to detect anti-TTR antibodies on both SF and sera of JIA patients and controls. A statistically significant antibody response to TTR was observed in both SF (Figure 4A and Supplemental Figure 4) and sera from JIA patients as compared with healthy controls (Figure 4B and Supplemental Figure 4). Additionally, JIA patients presented statistically significant increases in levels of TTR protein in both SF and sera (Figure 4, C and D). A positive correlation between TTR amount and anti-TTR antibody production was also observed (Figure 4E).

TTR has a natural tendency to misfold, aggregate, and precipitate $(38,39)$. Additionally, the oxidations we mapped by mass spectrometry on TTR could further increase its aggregation. Since protein aggregation can increase immunogenicity, we analyzed the extent of TTR aggregates in the SF. An equal amount of proteins from control and JIA SF were run on a native gel, and the membrane was blotted with anti-TTR antibodies. Physiologically, TTR is mostly present as a tetramer with a molecular weight of $60 \mathrm{kDa}$. In addition, complexes formed by two TTR tetramers could be observed in a native gel in all control patients (Figure 5A), whereas high-molecular-weight complexes with molecular weights from $240 \mathrm{kDa}$ up to 1,000 $\mathrm{kDa}$ could only be observed in the JIA SF (Figure 5A). Analysis by SDS-PAGE showed that all complexes separated into dimers and monomers, indicating that the TTR aggregates are mostly formed through noncovalent interactions (Figure 5B). 
A $\mathrm{kDa}$

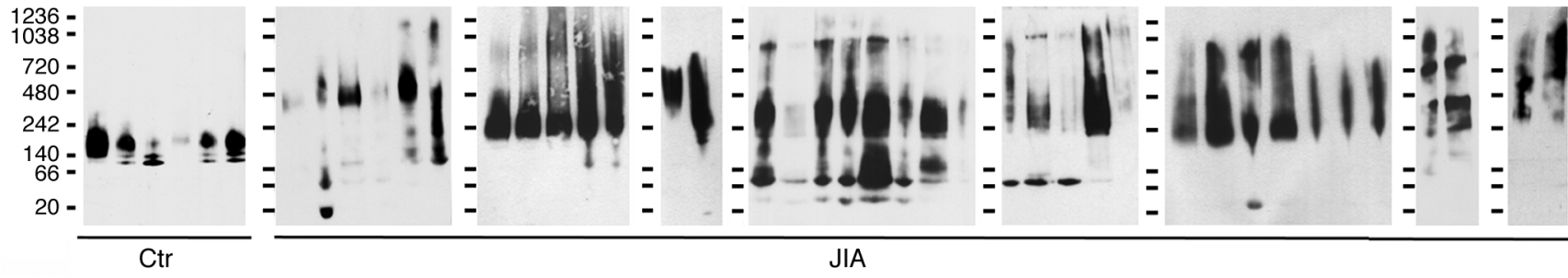

B $\mathrm{kDa}$

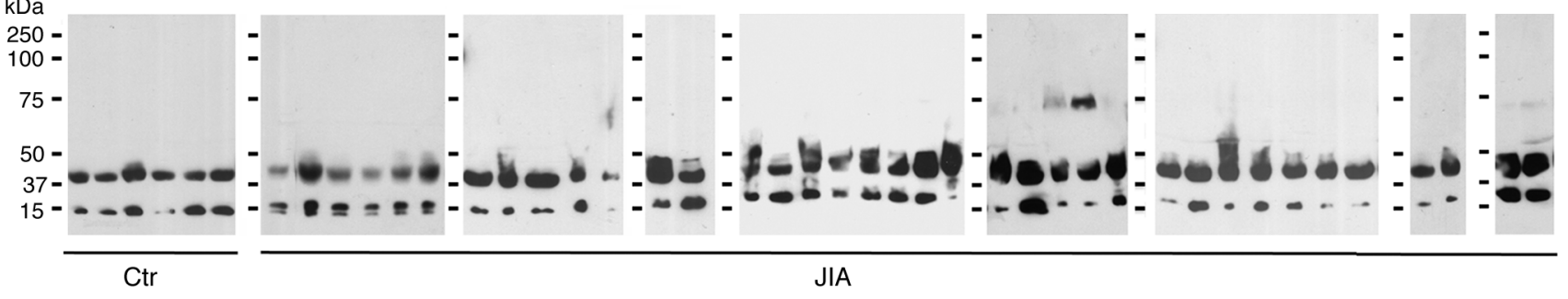

Figure 5. Transthyretin aggregates in the synovial fluid of juvenile idiopathic arthritis. Western blot analysis of transthyretin (TTR) proteins present in the synovial fluid of controls and juvenile idiopathic arthritis (JIA) patients run on a (A) native gel or (B) SDS-PAGE (representative gel out of 2 runs). TTR monomers $(15 \mathrm{kDa}$ ), dimers (30 kDa), tetramers $(60 \mathrm{kDa})$, and aggregates (above $60 \mathrm{kDa}$ ) are visible in JIA patients when run on a native gel. The patient ID for each lane is reported in Supplemental Figure 5.

$T$ cell responses to TTR protein and peptides. To further analyze the extent of the immune response to TTR, we tested T cell reactivity to the TTR peptides found in the peptidome/degradome of the JIA SF Four different TTR peptides, previously sequenced by MS/MS (Supplemental Table 3), were synthesized (Figure 6A). Since one of the peptides found in the SF presented extensive PTMs, the peptide was synthesized with or without the carbonyl groups previously mapped by mass spectrometry, including methionine oxidation and proline and lysine hydroxylation (Figure 6A). Mononuclear cells were gradient purified from the SF of 17 JIA patients (Supplemental Table 1) and stimulated in the presence or absence of different concentrations of TTR peptides. Due to the paucity in the number of T cells retrieved in the SF, the FACS analysis assessing IFN- $\gamma$, TNF- $\alpha$, and IL-17 production could be performed in all 17 patients, whereas T cell proliferation assay, as determined by CFSE labeling, was performed in 9 JIA patients (Supplemental Table 1 and Figures 6 and 7).

Cytokine responses were considered positive when a stimulation index (ratio between control and peptide-induced response) above 3 was observed. A markedly increased production of TNF- $\alpha$ and IFN- $\gamma$ was observed in the $\mathrm{CD}^{+} \mathrm{T}$ cell population of 3 out of $17 \mathrm{JIA}$ patients upon stimulation with one or more of the TTR peptides (Figure 6). No increase in IL-17 production could be detected (data not shown). Additionally, the two JIA patients with the highest increase in TNF- $\alpha$ and IFN- $\gamma$ production also had a modest T cell-proliferative response to the same TTR peptides, as detected by CFSE labeling (Figure 7). The patients with a positive response to the TTR peptides were clinically indistinguishable from the nonresponsive ones (Figures 6 and 7).

Since it was previously shown that a percentage of JIA patients express the DRB ${ }^{*} 0101$ haplotype, and one of the subjects responsive to the peptides was positive for DRB1*0101 (Supplemental Table 1), we tested peptide-binding affinity using recombinant HLA-DR1. Three out of four peptides bound HLA-DR1 with affinities within the low to high nanomolar range, similar to the HA peptide-positive control (Figure 8A).

To further analyze the HLA-DR1-restricted T cell response to TTR, we immunized HLA-DR1 humanized mice with the TTR peptides (Figure 8B), the native TTR protein, aggregated TTR, or aggregated/oxidized TTR to mimic the forms of the protein found in the SF (Figure $8 \mathrm{C}$ ). Proliferative responses were considered positive when a stimulation index (ratio between control and peptide-induced proliferative response) above 2.5 was observed. All tested peptides could be identified as cryptic or type B epitopes, as a statistically significant $\mathrm{T}$ cell-proliferative response could only be observed when mice were immunized with the peptides but not with the full-length native TTR (Figure 8C and ref. 40). Similar to native TTR, immunization with aggregated TTR did not induce a proliferative response to the cryptic peptides (Figure $8, \mathrm{~B}$ and $\mathrm{C}$ ). On the other hand, immunization with aggregated and oxidized TTR was able to induce a statistically significant proliferative response to two of the tested peptides (Figure $8 \mathrm{C}$ ). 
A

$\begin{array}{ll}\text { Peptides \# } & \text { No peptide } \\ 1 & \text { KCPLMVKVLDAVRGSPAIN } \\ 2 & \text { AVRGSPAINVAV }= \\ 3 & \text { KCPLMVKVLDAVRGSPAIN } \\ 4 & \text { AGPTGTGESKCPLMVKVLDAVRGSPAIN }\end{array}$

B

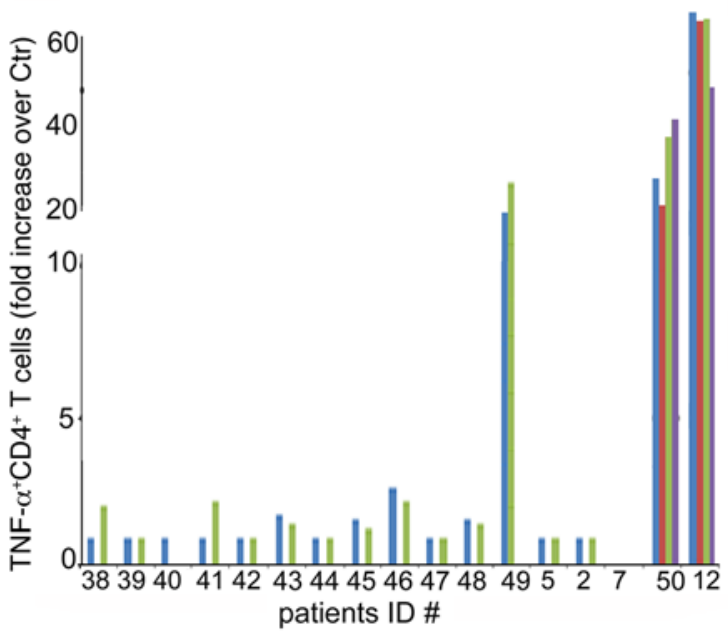

,

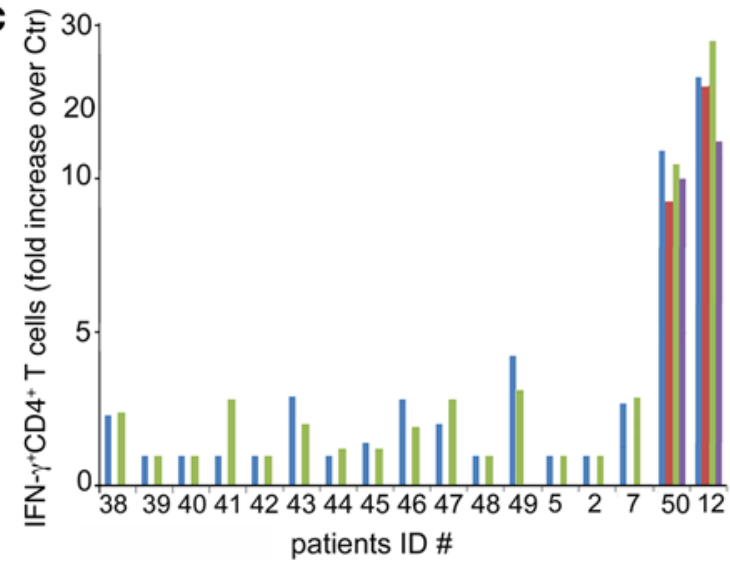

\section{D patients ID \#: 45}
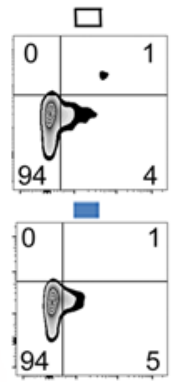

(1)
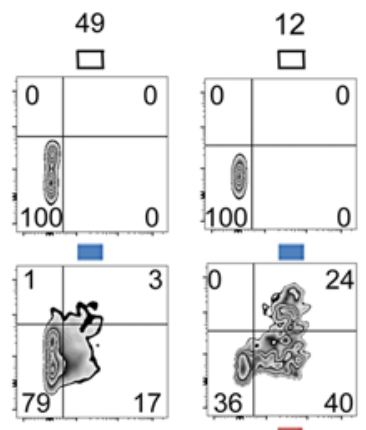

0
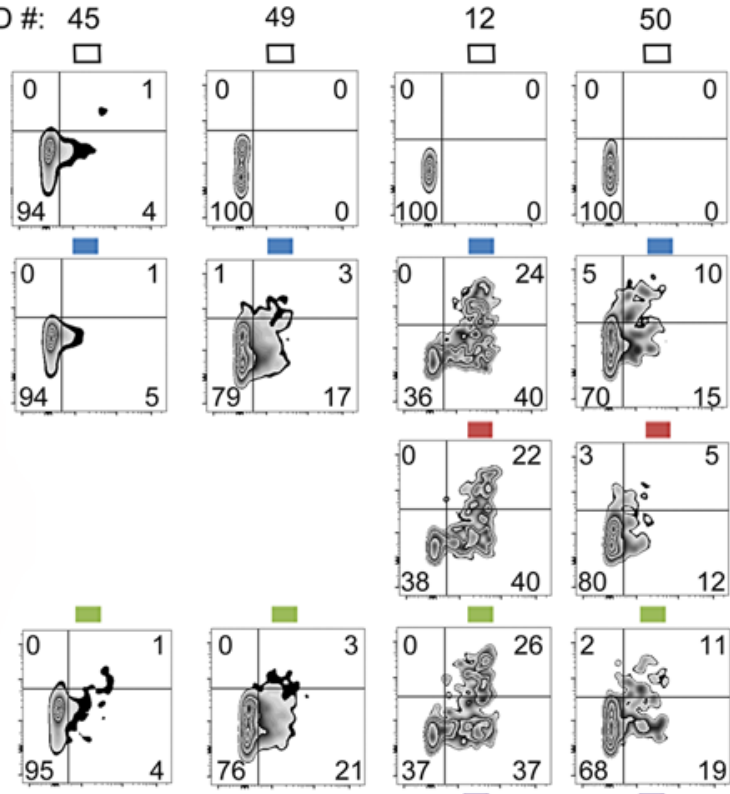

38
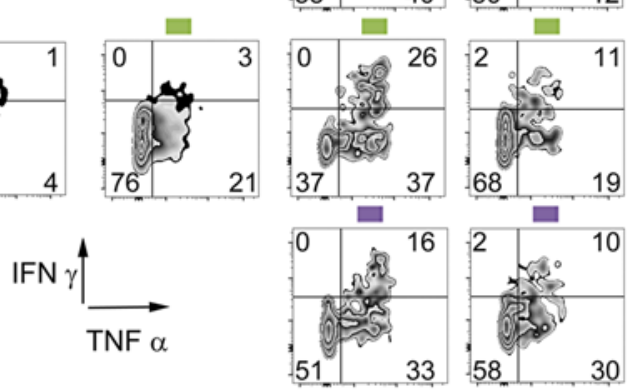

Figure 6. Production of TNF- $\alpha$ and IFN- $\gamma$ by T cells isolated from synovial fluid of juvenile idiopathic arthritis patients in response to transthyretin peptides. (A) Primary sequence of transthyretin (TTR) peptides. Residues in red are posttranslational modified ( $M$ is oxidized, and K and P are hydroxylated). (B) Fold increase of CD4+ $T$ cells producing TNF- $\alpha$ found in the synovial fluid (SF) of juvenile idiopathic arthritis (JIA) patients. Data are reported as fold increase in the number of TNF- $\alpha$-positive cells following stimulation with TTR peptides over unstimulated cells (1 out of 2 representative experiments is shown). (C) Fold increase of CD4+ $T$ cells producing IFN- $\gamma$ found in the SF of JIA patients (one out of two representative experiments is shown). Data are reported as fold increase in the number of IFN- $\gamma$-positive cells following stimulation with TTR peptides over unstimulated cells. (D) Examples of FACS analysis of TNF- $\alpha$ - and IFN- $\gamma$-producing T cells from different JIA patients before and after stimulation with TTR peptides. HLA-DR type of patient 12 is DRB1*03:07/07:01 and that of patient 50 is $\mathrm{DRB}^{*} 01: 01 / 11: 01$.

\section{Discussion}

The current study characterizes TTR as an autoantigen potentially involved in the pathogenesis of JIA. A statistically significant increase in the amount of TTR and anti-TTR antibody was observed in the SF and plasma of JIA patients as compared to controls. Proteomic analysis identified TTR protein among the IgGeluted antigens. Three HLA-DR1-restricted, naturally processed TTR epitopes, which induced CD4 ${ }^{+} \mathrm{T}$ cell expansion and IFN- $\gamma$ and TNF- $\alpha$ production in a subset of patients, were also identified.

TTR is a molecular chaperone that carries thyroxine, retinol (vitamin A), and several small molecules, including drugs, resveratrol, and toxins. TTR is synthesized in the liver, choroid plexus, and retinal epithelium and released in the serum and cerebrospinal fluid. Structurally, TTR can assemble as a dimer formed by two identical monomers of 127-amino acid residues or as a tetrameric structure formed by a face-to-face association of two dimers, which generate a quaternary complex of $60 \mathrm{kDa}$, with an inner channel for molecular transport. 


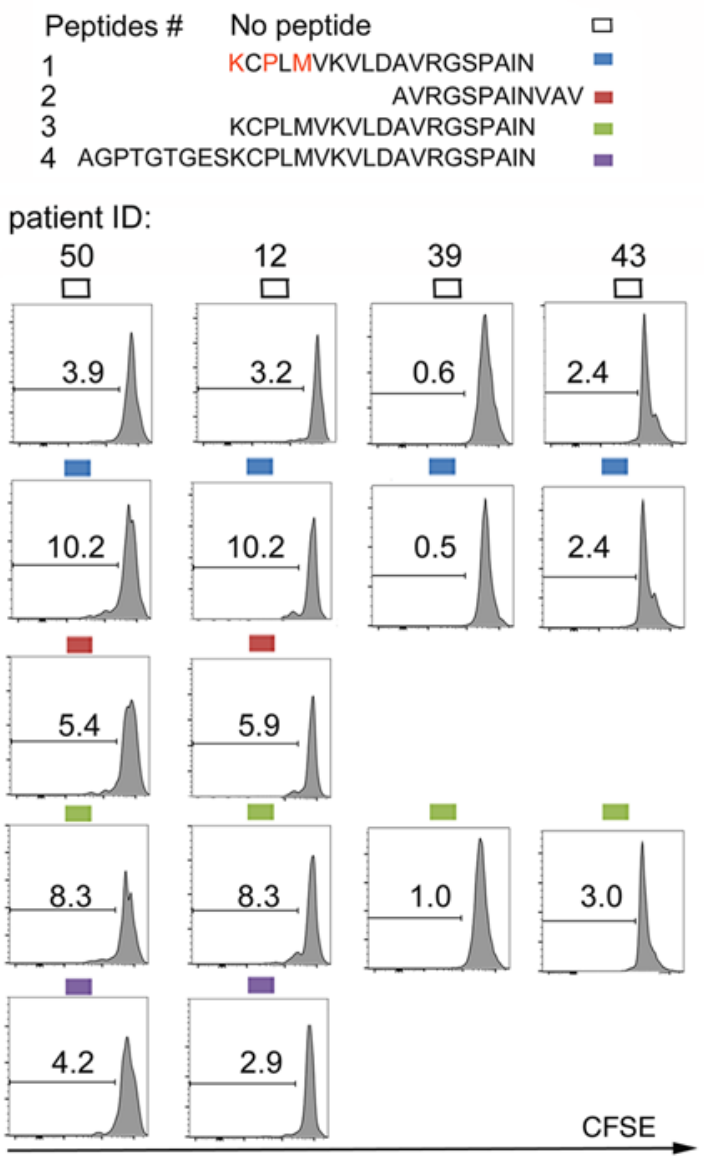

Figure 7. Proliferative responses of T cells isolated from synovial fluid of juvenile idiopathic arthritis patients after stimulation with transthyretin peptides. Histograms of CFSE-labeled T cells from different juvenile idiopathic arthritis (JIA) patients before and after stimulation with transthyretin peptides (1 out of 2 representative experiments is shown). HLA-DR type of patient 12 is $\mathrm{DRB} 1^{*} 03: 07 / 07: 01$ and that of patient 50 is DRB1*01:01/11:01.

TTR is particularly enriched in $\beta$ sheets and has a natural tendency to dissociate, unfold, and precipitate into amyloid structures. Indeed TTR is particularly enriched in senile systemic amyloidosis (38). Additionally, several TTR point mutations have been mapped to familial amyloid polyneuropathy and familial amyloid cardiomyopathy (41-43). In contrast to the senile form of amyloidosis, patients with TTR mutations are affected by amyloidosis much earlier in life (38).

The finding of TTR aggregates in the SF, synovial membrane, and eye $(22,26,39)$ and the finding that JIA affects young children led us to hypothesize that JIA could be another manifestation of a familial amyloidosis and could be associated with a point mutation specifically affecting the joints. Genome sequencing of 30 patients affected by JIA did not reveal mutation or allelic variants in any of the 4 exons of the gene encoding TTR. These data are in agreement with that reported by Takanashi, who sequenced this gene in 322 OA patients and did not find any evidence for mutant forms of TTR (25). However, as previously noted, TTR has a tendency to misfold and aggregate, thus promoting amyloidosis, even when nonmutated. During aggregation, the TTR tetramer dissociation is known to be rate limiting for amyloid fibril formation, albeit a partial misfolding of the monomer must also occur to induce amyloidogenesis (44-47).

We can envision several reasons why TTR would unfold and aggregate in JIA patients, even when nonmutated. As shown in our study, many JIA patients have significantly increased levels of TTR and anti-TTR antibodies in the SF as well as in the plasma $(23,24,48)$. Considering the tendency of TTR to precipitate and aggregate, even a small increase in TTR synthesis or half-life could easily bring the protein out of solution (44-46). In addition, the primary role of TTR as a chaperone in transport of small endogenous and exogenous molecules, including drugs and toxins, could favor TTR unfolding and aggregation (43). Furthermore, the SF is a highly viscous milieu, as compared to other biological fluids, and this high viscosity, which is associated with increased TTR production, could facilitate precipitation and aggregation $(8,37)$. Finally, inflammation is known to increase production of reactive oxygen and nitrogen species, which, in turn, can induce TTR oxidation, which by itself, is a known cause of protein aggregation (49).

Protein aggregates are known to be immunogenic through different mechanisms. Due to their high mul- 
A
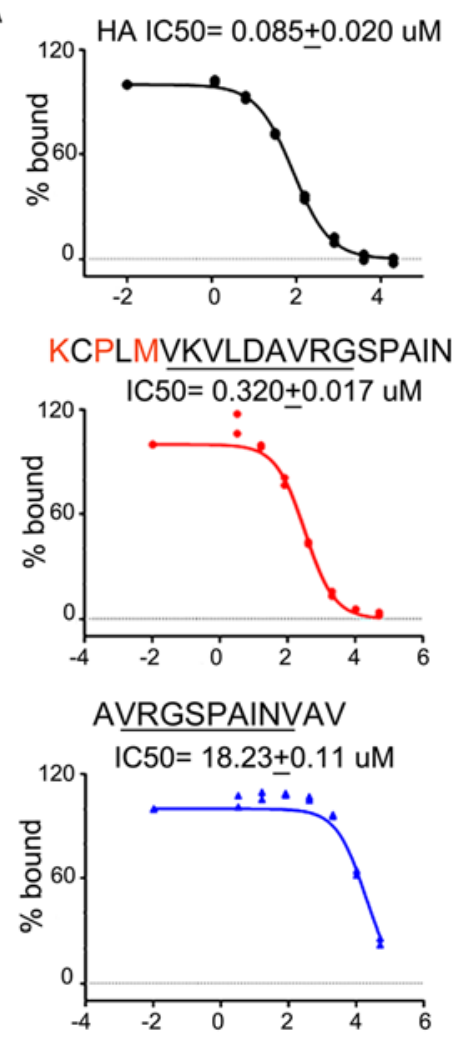

KCPLMVKVLDAVRGSPAIN

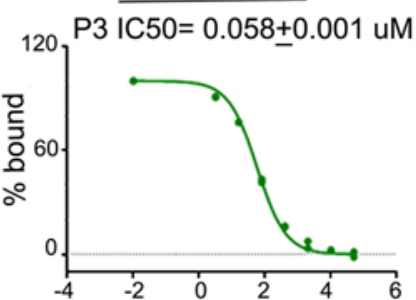

AGPTGTGESKCPLMVKVLDAVRGSPAIN

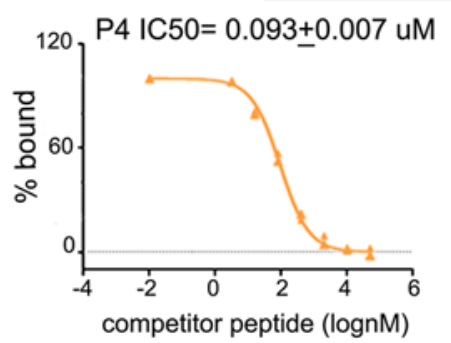

B

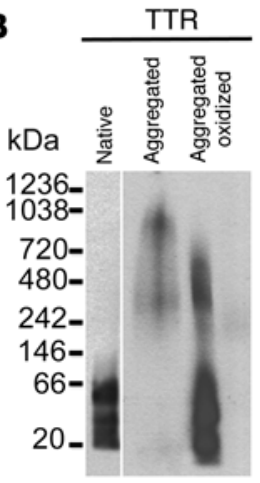

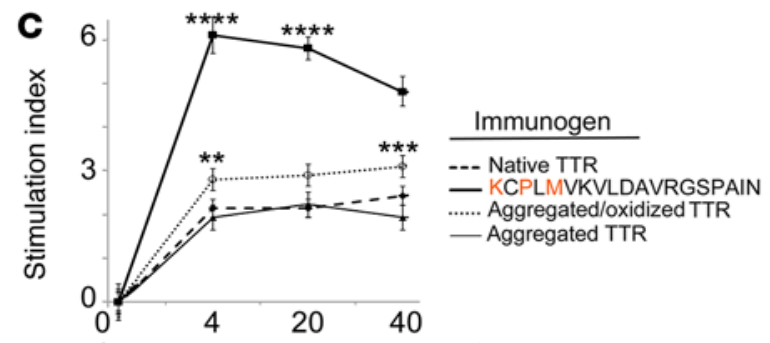

$\mu \mathrm{M}$ (KCPLMVKVLDAVRGSPAIN)
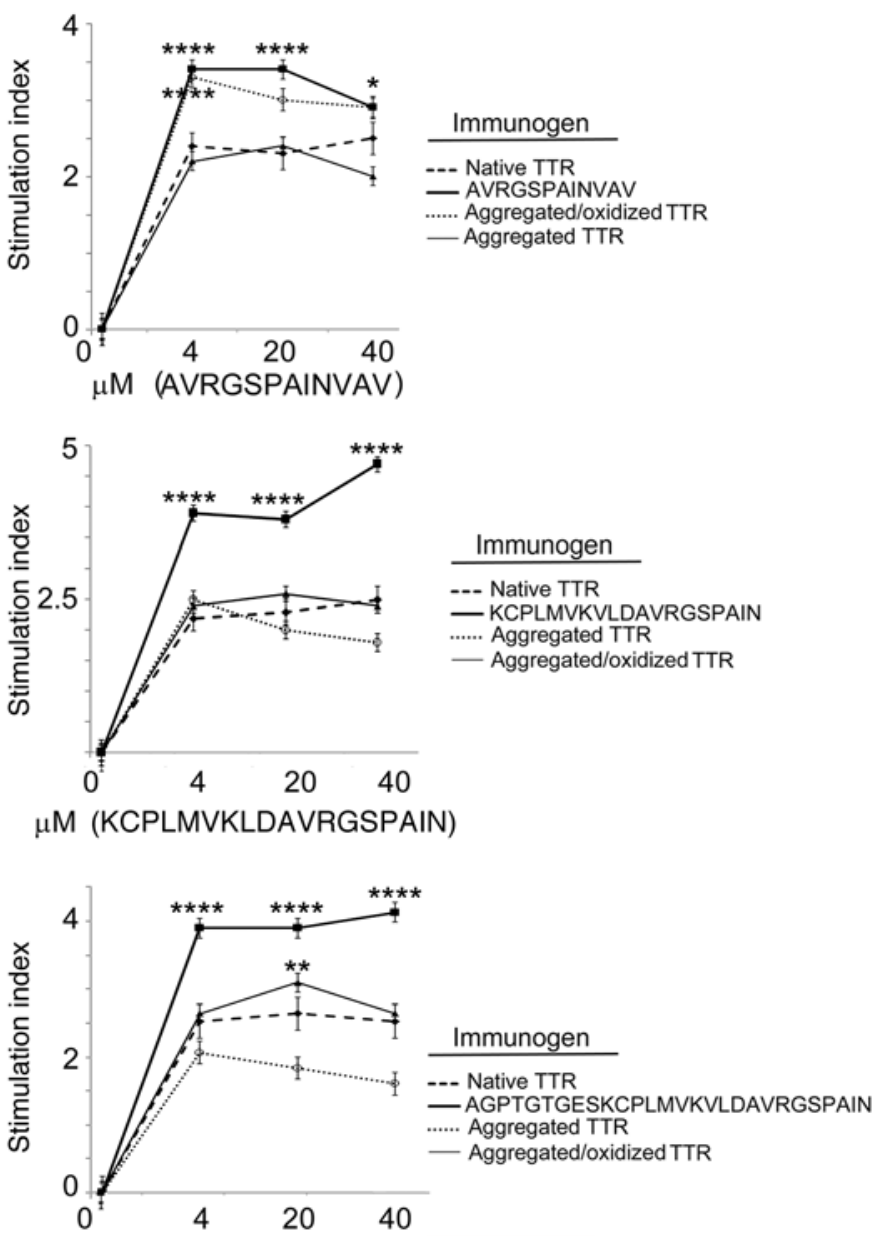

$\mu \mathrm{M}$ AGPTGTGESKCPLMVKVLDAVRGSPAIN

Figure 8. HLA-DR1-binding affinity and proliferative response to transthyretin peptides following immunization of HLA-DR1 mice with native, aggregated, or aggregated/oxidized transthyretin. (A) Inhibition binding curves (percentage bound test peptide vs. test peptide concentration [nM]) for transthyretin (TTR) peptides. Binding to HLA-DR1 molecules measured at 72 hours (1 out of 3 representative experiments is shown). Shown at the top of the graphs are the peptide sequences as well as the calculated values for binding $\left(\mathrm{IC}_{50}[\mu \mathrm{M}]\right)$. Binding of the immunodominant viral epitope $\mathrm{HA}$ peptide is shown as control. (B) Native gel showing native TTR (monomer at $15 \mathrm{kDa}$ and tetramer at $60 \mathrm{kDa}$ ); aggregated TTR, following exposure to low pH; and aggregated and oxidized TTR, following oxidation by Fenton reaction. One out of three gels is shown. (C) T cell-proliferative responses to increasing concentrations of the reported TTR peptides following immunization with native or aggregated or aggregated/oxidized TTR. HLA-DR1 mice were immunized as reported in Methods, popliteal and axillary nodes were harvested 3 weeks later, and T cells were rechallenged in vitro with increasing concentrations of the reported immunogens. Data are reported as stimulation index (BrDu incorporation following antigen stimulation over BrDu incorporation in absence of specific antigen). Data were compiled from 3 separate immunizations $(n=6)$. Experiments to evaluate T cell proliferation in response to antigen were run in quadruplicate for each antigen concentration tested. Mean \pm SD. Data were analyzed by 1 -way ANOVA $(P<0.005)$ and Tukey test. Asterisks indicate statistical significance, calculated at each concentration, between the peptide stimulation index and the native TTR or between the native TTR and the unfolded/carbonylated TTR. ${ }^{*} P<0.05 ;{ }^{* *} P<0.01 ;{ }^{* *} P<0.005 ;{ }^{* * *} P<0.001$. 
timerization, aggregates can cross-link BCRs and stimulate a downstream signaling cascade that induces B cell differentiation and IgG secretion in a T cell-dependent and/or -independent manner (50-53). Other reports indicate that highly ordered oligomerized antigens, as found in aggregates, activate the immune system, as these aggregated antigens resemble structures of pathogens and therefore can be recognized as PAMPs by pattern recognition receptors $(54,55)$. Moreover, protein aggregation has been shown to facilitate phagocytosis by antigen-presenting cells, which could potentially increase the antigenic load and number of MHC II epitopes presented to autoreactive T cells. Finally, protein aggregate can induce amyloid formation and direct complement activation (56).

The best clinical examples of protein aggregate-induced immunogenicity derive from the aggregation of therapeutic proteins (55), which, upon continuous injection, can induce an array of immune responses from the generation of autoantibody and delayed hypersensitivity to cytokine release syndrome and anaphylaxis (57). A second well-characterized example is islet amyloid polypeptide (IAPP), a pancreatic protein known to form amyloid fibrils in patients with type 2 diabetes. Aggregates of human IAPP, but not nonamyloidogenic IAPP, induce mRNA expression of different proinflammatory cytokines, including IL-1 $\beta$, TNF- $\alpha$, and IL- 6 , in pancreas resident macrophages.

PTMs, such as oxidation, are also known to induce protein immunogenicity by changing the structure and sequence of self epitopes (58). In our study, we mapped oxidized TTR epitopes in the SF of JIA patients, and, by immunizing transgenic HLA-DR1 mice with native, aggregated, or aggregated/oxidized TTR, we determined that protein oxidation enhances $\mathrm{T}$ cell responses. In our analysis, oxidation was the only modification associated with TTR found in the SF of JIA patients. However, we did find citrullination of fibrinogen, albumin, gelsolin, and A $\alpha$-2-HS-glycoprotein; citrullination of fibrinogen and $\alpha$ enolase were previously reported in JIA $(59,60)$. In general, carbonylation is a much more frequent PTM, which is often observed during chronic inflammation. Indeed, in contrast to citrullination, which requires enzymatic activity to modify arginine to citrulline, carbonylation is a nonenzymatic reaction, directly achieved by reactive oxygen species- and reactive nitrogen species-induced oxidative stress commonly present in acute and chronic inflammatory conditions.

In our analysis restricted to the HLA-DR1 haplotype, we observed a T cell response to 4 cryptic TTR peptides in only 3 out of 17 analyzed samples. However, the 4 peptides shared a common HLA-DR1-binding site, and 2 peptides shared the same primary amino acid sequence, with exception of PTMs. Thus, in the future, it will be important to map more TTR peptides with different HLA restriction and, more importantly, to find an immunodominant peptide driving the $\mathrm{T}$ cell immune response in different HLAsusceptible haplotypes.

Altogether, our data point to TTR as an autoantigen potentially involved in the pathogenesis of JIA and oxidation/aggregation of TTR as an important mechanism that facilitates TTR immunogenicity.

\section{Methods}

\section{Human subjects}

Fifty subjects with JIA and active arthritis of the knee requiring intra-articular corticosteroid injection were recruited from the pediatric rheumatology clinic at Montefiore Medical Center and Cincinnati Children's Hospital Medical Center (CCHMC). All patients met the International League Against Rheumatism criteria for JIA (61). Twenty-four subjects of between 3 and 18 years of age undergoing arthroscopic knee surgery for traumatic injuries were recruited as controls.

\section{Sample preparation}

SF was collected from controls and JIA patients and centrifuged at 1,500 $\mathrm{g}$ for 15 minutes at $4^{\circ} \mathrm{C}$ to remove cells. The supernatant was supplemented with a complete cocktail of protease inhibitors (Roche), filtered through $0.22-\mu \mathrm{m}$ Millipore filters, aliquoted, and stored at $-80^{\circ} \mathrm{C}$.

In the samples utilized for FACS analysis, lymphocytes present in the SF were isolated by gradient centrifugation with Ficoll-Hypaque (GE Healthcare). Between 6 and $15 \mathrm{ml}$ of SF was collected from each patient, and a range of 50,000 to 300,000 mononuclear cells per ml could be Ficoll-purified from the JIA SF.

Blood serum samples were prepared from Serum Separator Tubes according to the manufacturer's instructions (Becton Dickinson). 


\section{Chemicals}

Acetic acid (ULC/MS grade), acetonitrile, formic acid, methanol, trifluoroacetic acid (TFA), and ULC/ MS-grade water (for nanoLC analysis, 99\% purity) were purchased from Fisher Scientific. Dithiothreitol (DTT), iodoacetamide, ammonium bicarbonate, glycine, urea, thiourea, Coomassie Brilliant Blue R-250, $\mathrm{KCl}, \mathrm{KH}_{2} \mathrm{PO}_{4}, \mathrm{H}_{3} \mathrm{PO}_{4}$, and $\mathrm{Na}_{2} \mathrm{CO}_{3}$ were of the highest grade available from Sigma-Aldrich. Porcine trypsin $(20 \mu \mathrm{g}$, specific activity $>5,000$ units $/ \mathrm{mg}$ sequencing grade modified) was purchased from Promega. Complete TM proteinase inhibitor cocktail was purchased from Santa Cruz Biotechnology. The Aurum Serum protein Kit (catalog 732-6701) for depletion of IgG and albumin was purchased from Bio-Rad. The gel silver staining kit for mass spectrometry was from Pierce (catalog 24600). The OxiSelect Protein Carbonyl Spectrophotometric Assay kit (catalog STA-315) was from Cell Biolabs. SDS-PAGE precasted gels ( $4 \%-15 \%$ ) were from Bio-Rad (catalog $456-8083$ ). NativePAGE Novex $4 \%$ to $16 \%$ bis-Tris protein gels for blue-native electrophoresis of proteins and protein complexes (catalog BN1002BOX) and NativeMark unstained standards for size estimation of proteins in native PAGE (catalog LC0725) were purchased from Thermo Fisher Scientific, Life Sciences. Protein A/G Agarose (crosslinked 6\% beaded agarose, 20422) was purchased from Thermo Scientific Pierce. The purified native human prealbumin (TTR) (catalog 76000604) ( $96 \%$ purity by SDS-PAGE) was purchased from AbD Serotec (Bio-Rad). Monoclonal antibody 10E1 specific for human TTR (LF-MA0174) was purchased from Thermo Fisher Scientific, Life Sciences. Hyaluronidase from bovine testes (type IV-S, lyophilized powder) (catalog H3884-50MG) was purchased from Sigma-Aldrich. TMB substrate solution for ELISA (catalog N301) was purchased from Thermo Fisher Scientific. Collagenase type I (catalog 195109) was purchased from MP Biomedicals LLC. The Cell Proliferation ELISA BrdU (colorimetric) assay kit (catalog 11647229001) was purchased from Roche.

TTR peptides were synthesized at Karebay Biochem Inc. using standard solid-phase fluorenylmethyloxycarbonyl chemistry (HPLC-grade purity, $>95 \%$ ), as described previously (30). The following peptides were used in immunological assays: peptide $1: \mathrm{K}(+15.99) \mathrm{CP}(+15.99) \mathrm{LM}(+15.99)$ VKVLDAVRGSPAIN (where "+15.99" denotes the oxidation) (MW = 2, $058.2 \mathrm{~g} / \mathrm{mol}$ ); peptide 2: AVRGSPAINVAV (MW = $1,152.2 \mathrm{~g} / \mathrm{mol}$ ); peptide 3 : KCPLMVKVLDAVRGSPAIN (MW = 2,010.0 g/mol); and peptide 4: AGPTGTGESKCPLMVKVLDAVRGSPAIN (MW = 2,767.2 g/mol).

\section{D SDS-PAGE and in-gel trypsin digestion of the SF from JIA and control patients}

Prior to any experiment, $\mathrm{SFs}$ were digested with hyaluronidase $(30 \mathrm{kU} / \mathrm{ml})$ as previously described $(24)$ and depleted of albumins and IgG using Aurum Serum spin columns (Bio-Rad). Total protein concentration from human SF was determined using the Bradford assay (Bio-Rad).

Fifty $\mu$ g of each albumin- and IgG-depleted SF sample was run on a $4 \%$ to $20 \%$ SDS-PAGE as described previously for other biological fluids $(30,62,63)$. Gels were stained with Coomassie/Colloidal Brilliant Blue R-250, and 14 gel bands were cut across the whole lane. Gel pieces were washed 3 times with $25 \mathrm{mM} \mathrm{NH}_{4} \mathrm{HCO}_{3}$ in $50 \%$ acetonitrile, dried in a SpeedVac, reduced at $56^{\circ} \mathrm{C}$ for 1 hour with $10 \mathrm{mM}$ DTT in $25 \mathrm{mM} \mathrm{NH}_{4} \mathrm{HCO}_{3}$, and alkylated for 45 minutes at room temperature with $55 \mathrm{mM}$ iodoacetamide in 25 $\mathrm{mM} \mathrm{NH}_{4} \mathrm{HCO}_{3}$. Gel pieces were then washed with $25 \mathrm{mM} \mathrm{NH}_{4} \mathrm{HCO}_{3}$ for 10 minutes and dehydrated with $25 \mathrm{mM} \mathrm{NH}_{4} \mathrm{HCO}_{3}$ in $50 \%$ acetonitrile for 5 minutes. Following drying in a SpeedVac, the gel pieces were mixed with $12.5 \mathrm{ng} / \mu \mathrm{l}$ of trypsin and incubated on ice for 40 minutes in $25 \mathrm{mM} \mathrm{NH}_{4} \mathrm{HCO}_{3}$. Tryptic digestion was carried out at $37^{\circ} \mathrm{C}$ overnight. Tryptic peptides were extracted from the gel using $60 \%$ acetonitrile and $0.2 \%$ TFA. After evaporation, samples were desalted and concentrated with a C18 ZipTip (Millipore) and analyzed with a nanoLC-ESI-MS/MS (Ultimate Plus nano-HPLC system, LTQ, Thermo) interfaced with a TriVersa NanoMate nanoelectrospray ionization source (Advion BioSciences).

\section{Purification of the antigen-antibody complexes from the SF of JIA and controls patients}

Immune complexes were purified by immunoaffinity elution from the protein $A / G$ beads. Equal amounts of SF total proteome $(5 \mathrm{mg}$ ) were incubated with proteins $A$ and $G$ sepharose bead slurry overnight in TBS-Z buffer ( $50 \mathrm{mM}$ Tris- $\mathrm{HCl}$, pH 7.4, $150 \mathrm{mM} \mathrm{NaCl}, 0.05 \%$ sodium azide). Following incubation, the beads were collected and extensively washed with TBS-Z buffer, to remove any nonspecifically bound material, until the absorption at $280 \mathrm{~nm}$ fell below 0.05 absorption units. The antigens bound to the adsorbed IgG were eluted with $0.2 \mathrm{M}$ glycine/ $\mathrm{HCl}, \mathrm{pH} 2.5$ buffer. To remove free IgG from the resulting eluate, the specimen was passed through an ultrafiltration membrane with a 100-kDa cutoff device (Amicon). The protein eluates were further fractionated on a $4 \%$ to $20 \%$ SDS-PAGE gel followed by in-gel trypsin digestion and 
peptides extraction for MS/MS as described above.

\section{MS/MS sequencing of endogenous and tryptic peptides}

Equal amounts of $15 \mathrm{mg}$ of total protein from the SF of each of the JIA patients and controls were equilibrated in $1 \mathrm{ml}$ of sterile PBS buffer supplemented with a cocktail of protease inhibitors (Roche). Peptides were extracted using $0.2 \%$ TFA. Samples were then filtrated through a 10,000-Da cutoff filter device (Amicon) at $10^{\circ} \mathrm{C}$ for 30 minutes, desalted using pepClean C-18 spin columns (Pierce), and eluted with $70 \%$ acetonitrile containing $0.1 \%$ TFA.

Both naturally processed and eluted peptides as well as trypsin-digested peptides were subjected to nanoLC-MS/MS sequencing on a LTQ-Orbitrap Velos HR mass spectrometer using the combined HCD/ETD fragmentation method. In addition, each peptidome was sequenced on an ion trap mass spectrometer (LTQ, Thermo) using CID fragmentation. All samples were run in triplicate to perform label-free quantization.

\section{Protein database search of MS/MS spectra}

MS/MS raw spectra files were analyzed using two searching engines: Mascot version 2.3.02 (Matrix Science) and PEAKS DB versions 7.0 and 7.5 (Bioinformatic Solutions Inc.). The combined search was used to generate the final protein IDs (63).

\section{PTM analysis of the SF peptidome}

In the case of the peptidome sequenced on the LTQ-Orbitrap, we searched for additional chemical modifications, mainly those associated with inflammation and oxidative stress. Most of the variable chemical modifications included the amino acid oxidations reported by Mascot, mainly mono-oxidations on Arg, Cys (sulfenic acid), Phe, Lys, Met (Met-sulfoxide), Trp, and Tyr; di-oxidations on Arg, Cys (sulfinic acid), Met (Met-sulfone), Lys, Phe, Pro, Trp (N-formylkynurenine/dihydroxy Trp/dioxindolylalnine), and Tyr; tri-oxidations on Cys (cysteic acid); Arg-GluSa (glutamic semialdehyde conversion of Arg); and Pyrrolidinone (Pro). A list of all variable modifications used in the analysis of the peptidome is shown in Supplemental Table 2.

\section{Pathway and Gene Ontology term analysis}

Ingenuity pathways analysis software (Ingenuity Systems) was used to gain insight into the cellular location and molecular/cellular functional characteristics of the identified proteins listed in Supplemental Tables 1-5. An independent analysis of the biological pathways associated with each peptidome set was performed with the Database for Annotation, Visualization and Integrated Discovery v6.7 (http://www.genome. jp/kegg/pathway.html). In addition, the Generic Gene Ontology (GO) Term Mapper from Princeton. edu (http://go.princeton.edu/cgi-bin/GOTermMapper) was used to generate the GO terms associated with the global proteome depicted in Supplemental Table 1.

\section{MEROPS and CutDB analysis}

The SF peptidome from each JIA and control patient was used to infer the degradome, mainly by manual checking of the proteolytic cleave sites confined to each protein antigen substrate presented in Supplemental Table 2. The MEROPS database (http://merops.sanger.ac.uk/) (32) was searched in combination with the PMAP-CutDB proteolytic events database (http://cutdb.burnham.org/) (33) in order to assign the proteases families responsible for the generation of the $\mathrm{N}$-terminal and $\mathrm{C}$-terminal cleavage sites in the peptidomes.

\section{Heat map and protein cluster analysis of the peptidome and the immunoproteome}

Heat maps were generated using the quantitative data sets provided by the normalized spectral abundance factor values (average of 3 experiments), as calculated in Scaffold 4.0. The 3 independent analyses generated for the peptidomic profiling and the eluted immune complexes of each JIA patient were combined in one single biological sample using the MudPIT option in Scaffold 4 (Proteome Software), which was then contrasted against the JIA samples and the control MudPIT analyses. Following the $t$ test, Ward's dualclustering method was performed for comparison of controls (group 1) and JIA patients (group 2) using the ArrayTrack platform (US FDA, http://www.fda.gov/ScienceResearch/BioinformaticsTools/Arraytrack/). 
Spectrophotometric determination of carbonyl content in JIA and control SF

The carbonyl content (mainly carbonylated Pro, Arg, Lys, and Thr residues) of the total proteome from the SF of JIA and control patients was determined spectrophotometrically with the OxiSelect Protein Carbonyl Spectrophotometric Assay kit (Cell Biolabs) following the manufacturer's instructions. The absorbance of the DNPH-derivatized carbonyl groups was read at $375 \mathrm{~nm}$. A 1-cm-wide cuvette was used for all readings, and the final results were normalized to the total protein in each sample as nanomoles/mg protein. The samples were prepared in triplicate, and the average readings \pm SD were analyzed by 1 -way ANOVA $(P<0.05)$ followed by Tukey test.

Native blue gel electrophoresis, SDS-PAGE, and Western Blot analysis of TTR in SF Native blue gel electrophoresis. Equal volumes (10 $\mu \mathrm{l}$ from each SF sample) were mixed with the NativePAGE sample buffer (4X) and run on NativePAGE Novex $4 \%$ to $16 \%$ bis-Tris protein gels for 90 minutes at a $150-\mathrm{V}$ constant voltage. The gels were equilibrated in the transfer buffer ( $25 \mathrm{mM}$ Tris, $192 \mathrm{mM}$ glycine, 20\% (v/v) methanol, $\mathrm{pH} 8.3$ ) 2 times for 5 minutes each time before transfer onto the nitrocellulose membrane (Millipore) using Western blot procedure.

$S D S$-PAGE gel electrophoresis. Equal volumes (10 $\mu 1$ from each SF sample) were mixed with the sample buffer (containing both the SDS and the reducing agent, $\beta$-mercaptoethanol), heated at $95^{\circ} \mathrm{C}$ for 5 minutes, and run on $4 \%$ to $20 \%$ SDS-PAGE. Proteins were transferred to the nitrocellulose membrane $(0.45 \mu \mathrm{m}$, Millipore) using Western blot procedures.

Western blot analysis. Membranes were blocked using 5\% bovine serum albumin (BSA; Sigma-Aldrich) for 1 hour at room temperature. The membranes were then incubated with anti-human TTR (clone 10E1) (1:1,000 dilution) overnight at $4^{\circ} \mathrm{C}$ followed by HRP-conjugated anti-mouse antibody at room temperature with for 2 hours. Enhanced chemiluminescence assay (Thermo Scientific, Pierce) was used to develop the membranes.

Densitometry analysis of Western blots from the native gels was performed with the ImageJ 1.49 software. Macromolecular complexes containing TTR with MW $>240 \mathrm{kDa}$ were quantified, and their total densities were correlated with the anti-TTR antibodies and with the total TTR in the SF of all 50 tested patients. The Spearman $\mathrm{r}$ statistical analysis was used to determine the significance for all the correlations.

\section{Anti-TTR antibodies ELISA}

Each well of a 96-well ELISA plate (Nunc MaxiSorp) was coated with purified human TTR protein $(0.20$ $\mu \mathrm{g} / 100 \mu \mathrm{l}$ buffer containing $0.01 \mathrm{M} \mathrm{Na}_{2} \mathrm{CO}_{3}$ and $0.035 \mathrm{M} \mathrm{NaHCO}_{3}, \mathrm{pH} 9.6$ ). The plates were kept at $4^{\circ} \mathrm{C}$ overnight and washed 3 times with $200 \mu \mathrm{l}$ /well PBS with $0.05 \%$ Tween 20 (PBST), followed by blocking with $200 \mu \mathrm{l}$ /well of blocking buffer ( $2 \%$ BSA in PBS) for 2 hours at room temperature. Plates were then washed and incubated with $100 \mu \mathrm{l} /$ well of sera or SF (1:200 dilutions) for 3 hours at room temperature (in quadruple replicates). The plates were then washed 3 times with PBST and incubated with $100 \mu 1 /$ well of goat anti-human HRP conjugate for 2 hours at room temperature. The plates were developed with the TMB substrate solution, and the absorbance was read at $450 \mathrm{~nm}$ on the ELISA plate reader (Spectramax Plus; Molecular Devices).

\section{Anti-TTR ELISA}

Sera and SF samples were diluted 1:200 in coating buffer (0.01 $\mathrm{M} \mathrm{Na}_{2} \mathrm{CO}_{3}$ and 0.035 $\mathrm{M} \mathrm{NaHCO}_{3}, \mathrm{pH}$ 9.6) and incubated $\mathrm{ON}$ at $4^{\circ} \mathrm{C}$ in 96-well plates (Nunc MaxiSorp). The next day, the plates were washed 3 times with $100 \mu \mathrm{PBST} /$ well and incubated with $100 \mu \mathrm{l}$ blocking buffer (1\% BSA in 1X PBS) for 1 hour at room temperature. Plates were then washed with PBST and incubated with $100 \mu 1 /$ well of anti-TTR antibody $(1: 2,000)$ (anti-human TTR clone 10E1) for 2 hours at room temperature followed by the secondary antibody anti-mouse HRP conjugate (1:1,000 dilution) for 2 hours. Plates were developed with TMB solution. Each sample was run in quadruple replicates.

\section{$\mathrm{H}_{2} \mathrm{O}_{2}$ oxidation of TTR}

Human TTR ( $2 \mathrm{mg} / \mathrm{ml}$ in PBS buffer, $\mathrm{pH} 7.4$ ) was incubated with $3 \%$ hydrogen peroxide $\left(\mathrm{H}_{2} \mathrm{O}_{2}, \mathrm{H} 1009\right.$, Sigma-Aldrich) at room temperature for 30 minutes as previously described $(36,49)$. Oxidant was then removed through PBS exchange by filtration through Centriplus (Amicon) centrifugal filter devices $(3,000$ Da cutoff) at room temperature. The $\mathrm{H}_{2} \mathrm{O}_{2}$-induced oxidation and unfolding of TTR was monitored by 
native blue gel electrophoresis as described above.

\section{Acid-mediated TTR aggregation}

Acid-mediated aggregation and fibril formation experiments were carried out as described previously (64). Briefly, human TTR in PBS buffer $(0.5 \mathrm{mg} / \mathrm{ml})$ was diluted $1: 1$ with acetate buffer $(200 \mathrm{mM}$ sodium acetate, $\mathrm{pH} 4.2,100 \mathrm{mM} \mathrm{KCl}, 1 \mathrm{mM}$ EDTA) to achieve a final $\mathrm{pH}$ of 4.4. The TTR solution was incubated at $37^{\circ} \mathrm{C}$ for 24 hours. The acid-induced unfolding and aggregation of TTR was monitored by native blue gel electrophoresis as described above. Additionally, the acid-induced TTR aggregation was monitored by increased turbidity at $400 \mathrm{~nm}$.

\section{HLA-DR1-binding affinity}

$\mathrm{IC}_{50}$ was measured by fluorescence polarization (FP) assay as previously described (65). Soluble recombinant MHC class II molecules (HLA-DRA*01:01; DRB1*01:01) were expressed in Drosophila S2 cells and purified by immunoaffinity chromatography. N-terminally acetylated influenza hemagglutinin HA306318 analog (Ac-PRFVKQNTLRLAT) (21st Century Biochemicals) was labeled with Alexa488 tetrafluorophenyl ester (Invitrogen) using the lysine in the P3 position and purified by Jupiter C18 reverse-phase chromatography (Phenomenex); it was then used as probe peptide in a competition assay. DR1 (100 nM) was incubated with probe peptide Alexa488-HA306-318 (25 nM), together with a series of 5-fold dilutions of test peptides, starting with $50 \mu \mathrm{M}$. Competition of each concentration of test peptide with probe Alexa488-HA306-318 for binding to DR1 was measured by FP after incubation at $37^{\circ} \mathrm{C}$ for 72 hours (1). FP values were converted to percentage bound values as (FP_sample - FP_free)/(FP_no_comp - FP_free), where FP_sample is the FP value for sample well; FP_free is the FP value for free Alexa488-HA306-318; and FP_no_comp is the FP value for wells without competitor peptides. Percentage bound peptide was plotted versus concentration of test peptide and fit the curve to equation $y=1 /\left(1+[\mathrm{pep}] / \mathrm{IC}_{50}\right)$, where [pep] is the concentration of test peptide, $y$ is the percentage of probe peptide bound at that concentration of test peptide, and $\mathrm{IC}_{50}$ is the $50 \%$ inhibition concentration of the test peptide.

\section{HLA genotyping}

HLA class I (A, B, and C) and class II (DRB1, DQA1, DQB1, DPA1, and DPB1) typing were derived from CCHMC pediatric biorepository data. Genotyping was performed using PCR as described previously (15). Data analysis is reported in Supplemental Figure 6.

\section{Flow cytometry and cytokine detection}

Five hundred thousand SF mononuclear cells (SFMC) were labeled with the CellTrace CFSE Cell Proliferation Kit according to manufacturer's instructions (Life Technologies). SFMC were stimulated in the presence or absence of TTR peptide for 3 days at $37^{\circ} \mathrm{C}, 5 \% \mathrm{CO}_{2}$, and restimulated for 4 hours with the same peptide and Brefeldin A (10 $\mu \mathrm{g} / \mathrm{ml}$, eBioscience). Cells were fixed with Fix/Perm buffer (eBioscience) and stained with the following monoclonal antibodies against human proteins: PeCy7-conjugated anti-IFN- $\gamma$, (eBioscience), AF700-conjugated anti-TNF- $\alpha$ (BioLegend), and BV500-conjugated anti-CD4 (BD Bioscience). Dead cells were excluded using Live/Dead fixable dye according to the manufacturer's instructions (Life Technologies). Flow cytometry data were acquired on a BD LSR Fortessa and analyzed with FlowJo v10 software (FlowJo).

\section{TTR protein and TTR peptides immunization}

Three preparations of TTR proteins were used for immunization: (a) native; (b) $\mathrm{H}_{2} \mathrm{O}_{2}$-induced oxidized and unfolded; and (c) acid-induced unfolded and aggregated. Mice were immunized with TTR protein solution or TTR peptides ( $100 \mu \mathrm{g}$ in $100 \mu \mathrm{l}$ of PBS, emulsified with $100 \mu 1$ of complete Freund's adjuvant) at the base of the tail and back of the neck. Twenty-one days after immunization mice were sacrificed and inguinal and axillary lymph nodes were collected.

\section{T cell proliferation}

The lymph nodes from the immunized mice were collected in sterile Hank's solution and incubated in $1 \mathrm{ml}$ of collagenase type $\mathrm{I}(1 \mathrm{mg} / \mathrm{ml})$ at $37^{\circ} \mathrm{C}$ for 15 minutes, and a single-cell suspension was prepared through a $100-\mu \mathrm{m}$ cell strainer. Cells were washed twice in complete DMEM and seeded at $4 \times 10^{5}$ cells $/ 100 \mu 1 /$ 
well in MICROTEST U-Bottom 96-well polystyrene sterile plates. Cells were incubated with or without increasing concentrations of the TTR peptides $(4,20,40$, and $80 \mu \mathrm{M})$ or the native or aggregated or carbonylated TTR protein solutions $(25,50,100$, and $200 \mu \mathrm{g} / \mathrm{ml})$ in quadruple replicates. Cells were incubated for 3 days at $37^{\circ} \mathrm{C}$ and supplemented with $5 \% \mathrm{CO}_{2}$. Cell proliferation was measured on the fourth day using BrdU-labeling reagent (11647229001; Roche).

\section{Statistics}

Statistical analysis was performed using Windows GraphPad Prism 6 (GraphPad Software). Comparisons of the expression of human TTR antigen and anti-TTR autoantibodies between healthy controls and JIA patients were performed using the 2-tailed paired $t$ test. $\mathrm{T}$ cell proliferation data were analyzed using the 2-tailed unpaired 1-way ANOVA. A $P$ value less than 0.05 was considered significant.

\section{Study approval}

For the human study, risks and benefits were reviewed with and consent was obtained from participants. Experimental procedures were approved by the Internal Review Boards of the Children's Hospital at Montefiore, the Albert Einstein College of Medicine, and the CCHMC. The experimental mouse protocol was approved by the committee at Albert Einstein College of Medicine.

\section{Author contributions}

CCC, HM, AL, GJ, AB, FB, FAS, GP, and CM performed the experiments. CCC, HM, AB, CM, LJS, and LS analyzed the data. GP, NC, and NI provided reagents. CCC, HM, GJ, JH, SAP, and LS wrote the manuscript.

\section{Acknowledgments}

This work was supported by NIH grants AG045223 (to L. Santambrogio), AI38996 (to L.J. Stern), and AI48833 (to L.J. Stern). We acknowledge the Laboratory for Macromolecular Analysis and Proteomics (NIH grant 1S10RR019352) at Albert Einstein College of Medicine. We thank Monica Tsoras of the CCHMC Pediatric Rheumatology Tissue Core for excellent technical support and database management. This research was supported in part by the Cincinnati Children's Research Foundation and its Cincinnati Genomic Control Cohort. All flow cytometry data were acquired using equipment maintained by the Research Flow Cytometry Core in the Division of Rheumatology at CCHMC, supported in part by NIH grants AR-47363, DK78392, and DK90971. H. Moncrieffe was supported in part by NIH grants AR-47363 and AR-48929.

Address correspondence to: Laura Santambrogio, Department of Pathology, Albert Einstein College of Medicine, 1300 Morris Park Avenue, Forchheimer Building Room 140, Bronx, New York, New York 10461, USA. Phone: 718.430.3458; E-mail: laura.santambrogio@einstein.yu.edu.

1. Alexeeva EI, et al. Efficacy and safety of repeat courses of rituximab treatment in patients with severe refractory juvenile idiopathic arthritis. Clin Rheumatol. 2011;30(9):1163-1172.

2. Barnes MG, et al. Biologic similarities based on age at onset in oligoarticular and polyarticular subtypes of juvenile idiopathic arthritis. Arthritis Rheum. 2010;62(11):3249-3258.

3. Finnegan S, et al. Synovial membrane protein expression differs between juvenile idiopathic arthritis subtypes in early disease. Arthritis Res Ther. 2014;16(1):R8.

4. Gibson DS, et al. Proteomic analysis of recurrent joint inflammation in juvenile idiopathic arthritis. J Proteome Res. 2006;5(8):1988-1995.

5. Gibson DS, Qiu J, Mendoza EA, Barker K, Rooney ME, LaBaer J. Circulating and synovial antibody profiling of juvenile arthritis patients by nucleic acid programmable protein arrays. Arthritis Res Ther. 2012;14(2):R77.

6. Gorelik M, et al. Follistatin-like protein 1 and the ferritin/erythrocyte sedimentation rate ratio are potential biomarkers for dysregulated gene expression and macrophage activation syndrome in systemic juvenile idiopathic arthritis. J Rheumatol. 2013;40(7):1191-1199.

7. Heiligenhaus A, Heinz C, Edelsten C, Kotaniemi K, Minden K. Review for disease of the year: epidemiology of juvenile idiopathic arthritis and its associated uveitis: the probable risk factors. Ocul Immunol Inflamm. 2013;21(3):180-191.

8. Kamphorst JJ, et al. Profiling of endogenous peptides in human synovial fluid by NanoLC-MS: method validation and peptide identification. J Proteome Res. 2007;6(11):4388-4396.

9. Lin YT, Wang CT, Gershwin ME, Chiang BL. The pathogenesis of oligoarticular/polyarticular vs systemic juvenile idiopathic arthritis. Autoimmunity reviews. 2011;10(8):482-489.

10. Low JM, et al. Proteomic analysis of circulating immune complexes in juvenile idiopathic arthritis reveals disease-associated proteins. Proteomics Clin Appl. 2009;3(7):829-840. 
11. Rosenkranz ME, et al. Synovial fluid proteins differentiate between the subtypes of juvenile idiopathic arthritis. Arthritis Rheum 2010;62(6):1813-1823.

12. Thompson SD, Barnes MG, Griffin TA, Grom AA, Glass DN. Heterogeneity in juvenile idiopathic arthritis: impact of molecular profiling based on DNA polymorphism and gene expression patterns. Arthritis Rheum. 2010;62(9):2611-2615.

13. Lin YT, Wang CT, Gershwin ME, Chiang BL. The pathogenesis of oligoarticular/polyarticular vs systemic juvenile idiopathic arthritis. Autoimmun Rev. 2011;10(8):482-489.

14. Prakken B, Albani S, Martini A. Juvenile idiopathic arthritis. Lancet. 2011;377(9783):2138-2149.

15. Hollenbach JA, et al. Juvenile idiopathic arthritis and HLA class I and class II interactions and age-at-onset effects. Arthritis Rheum. 2010;62(6):1781-1791.

16. Nistala K, et al. Th17 plasticity in human autoimmune arthritis is driven by the inflammatory environment. Proc Natl Acad Sci US A. 2010;107(33):14751-14756

17. Nistala K, Wedderburn LR. Th17 and regulatory T cells: rebalancing pro- and anti-inflammatory forces in autoimmune arthritis. Rheumatology. 2009;48(6):602-606.

18. Hinks A, et al. Dense genotyping of immune-related disease regions identifies 14 new susceptibility loci for juvenile idiopathic arthritis. Nat Genet. 2013;45(6):664-669.

19. Alexeeva EI, et al. Efficacy safety of repeat courses of rituximab treatment in patients with severe refractory juvenile idiopathic arthritis. Clin Rheumatol. 2011;30(9):1163-1172.

20. Heiligenhaus A, Miserocchi E, Heinz C, Gerloni V, Kotaniemi K. Treatment of severe uveitis associated with juvenile idiopathic arthritis with anti-CD20 monoclonal antibody (rituximab). Rheumatology (Oxford). 2011;50(8):1390-1394

21. Barnes MG, et al. Biologic similarities based on age at onset in oligoarticular and polyarticular subtypes of juvenile idiopathic arthritis. Arthritis Rheum. 2010;62(11):3249-3258.

22. Akasaki Y, et al. Transthyretin deposition in articular cartilage: a novel mechanism in the pathogenesis of osteoarthritis. Arthritis Rheumatol. 2015;67(8):2097-2107.

23. Ni M, et al. Transthyretin as a potential serological marker for the diagnosis of patients with early rheumatoid arthritis. Clin Exp Rheumatol. 2013;31(3):394-399.

24. Sharma S, et al. Identification of autoantibodies against transthyretin for the screening and diagnosis of rheumatoid arthritis PLoS One. 2014;9(4):e93905.

25. Takanashi T, et al. Synovial deposition of wild-type transthyretin-derived amyloid in knee joint osteoarthritis patients. Amyloid. 2013;20(3):151-155

26. Kalinina Ayuso V, et al. Intraocular biomarker identification in uveitis associated with juvenile idiopathic arthritis. Invest Ophthalmol Vis Sci. 2013;54(5):3709-3720.

27. Biomarkers for Juvenile Idiopathic Arthritis. Biomarker profiles Web site. http://www.biomarkerprofiles.com/juvenile_idiopathic_arthritis.html. Accessed on February 12, 2016.

28. Ling XB, et al. Urine peptidomic and targeted plasma protein analyses in the diagnosis and monitoring of systemic juvenile idiopathic arthritis. Clin Proteomics. 2010;6(4):175-193.

29. James EA, et al. HLA-DR1001 presents "altered-self" peptides derived from joint-associated proteins by accepting citrulline in three of its binding pockets. Arthritis Rheum. 2010;62(10):2909-2918.

30. Clement CC, et al. An expanded self-antigen peptidome is carried by the human lymph as compared to the plasma. PLoS One. 2010;5(3):e9863

31. Consolaro A, Varnier GC, Martini A, Ravelli A. Advances in biomarkers for paediatric rheumatic diseases. Nat Rev Rheumatol. 2015;11(5):265-275

32. Rawlings ND, Morton FR, Barrett AJ. MEROPS: the peptidase database. Nucleic Acids Res. 2006;34(Database issue):D270-D272.

33. Igarashi Y, et al. CutDB: a proteolytic event database. Nucleic Acids Res. 2007;35(Database issue):D546-D549.

34. Cannizzo ES, Clement CC, Sahu R, Follo C, Santambrogio L. Oxidative stress, inflamm-aging and immunosenescence. J Proteomics. 2011;74(11):2313-2323.

35. Cannizzo ES, et al. Age-related oxidative stress compromises endosomal proteostasis. Cell Rep. 2012;2(1):136-149.

36. Scharf B, et al. Age-related carbonylation of fibrocartilage structural proteins drives tissue degenerative modification. Chem Biol 2013;20(7):922-934

37. Raijmakers R, et al. Elevated levels of fibrinogen-derived endogenous citrullinated peptides in synovial fluid of rheumatoid arthritis patients. Arthritis Res Ther. 2012;14(3):R114.

38. Buxbaum JN. The systemic amyloidoses. Curr Opin Rheumatol. 2004;16(1):67-75

39. M'Bappe P, Grateau G. Osteo-articular manifestations of amyloidosis. Best Pract Res Clin Rheumatol. 2012;26(4):459-475

40. Reis e Sousa C, Unanue ER. Antigen processing. Curr Opin Immunol. 2014;26:138-139.

41. Levy J, Hawkins PN, Rowczenio D, Godfrey T, Stawell R, Zamir E. Familial amyloid polyneuropathy associated with the novel transthyretin variant Arg34Gly. Amyloid. 2012;19(4):201-203.

42. Jacobson DR, et al. Variant-sequence transthyretin (isoleucine 122) in late-onset cardiac amyloidosis in black Americans. $N E n g l$ JMed. 1997;336(7):466-473.

43. Saraiva MJ. Transthyretin mutations in health and disease. Hum Mutat. 1995;5(3):191-196

44. Colon W, Kelly JW. Partial denaturation of transthyretin is sufficient for amyloid fibril formation in vitro. Biochemistry. 1992;31(36):8654-8660

45. Lai Z, Colon W, Kelly JW. The acid-mediated denaturation pathway of transthyretin yields a conformational intermediate that can self-assemble into amyloid. Biochemistry. 1996;35(20):6470-6482.

46. Hammarstrom P, Wiseman RL, Powers ET, Kelly JW. Prevention of transthyretin amyloid disease by changing protein misfolding energetics. Science. 2003;299(5607):713-716

47. Sekijima Y, et al. The biological and chemical basis for tissue-selective amyloid disease. Cell. 2005;121(1):73-85

48. Naishiro Y, et al. [Plasma analysis of rheumatoid arthritis by SELDI]. Jpn J Clin Immunol. 2007;30(3):145-150.

49. Zhao L, Buxbaum JN, Reixach N. Age-related oxidative modifications of transthyretin modulate its amyloidogenicity. Biochemistry. 2013;52(11):1913-1926. 
50. Bachmann MF, Zinkernagel RM. Neutralizing antiviral B cell responses. Annu Rev Immunol. 1997;15:235-270.

51. Sauerborn M, Brinks V, Jiskoot W, Schellekens H. Immunological mechanism underlying the immune response to recombinant human protein therapeutics. Trends Pharmacol Sci. 2010;31(2):53-59.

52. Hunziker L, et al. Hypergammaglobulinemia and autoantibody induction mechanisms in viral infections. Nat Immunol. 2003;4(4):343-349.

53. Soulas $\mathrm{P}$, et al. Autoantigen, innate immunity, and $\mathrm{T}$ cells cooperate to break B cell tolerance during bacterial infection. $J$ Clin Invest. 2005;115(8):2257-2267.

54. Hermeling S, Crommelin DJ, Schellekens H, Jiskoot W. Structure-immunogenicity relationships of therapeutic proteins. Pharm Res. 2004;21(6):897-903.

55. Rosenberg SA, et al. Altered CD8(+) T-cell responses when immunizing with multiepitope peptide vaccines. J Immunother. 2006;29(2):224-231.

56. Rogers J, et al. Complement activation by beta-amyloid in Alzheimer disease. Proc Natl Acad Sci U S A. 1992;89(21):10016-10020.

57. Stebbings R, et al. "Cytokine storm" in the phase I trial of monoclonal antibody TGN1412: better understanding the causes to improve preclinical testing of immunotherapeutics. J Immunol. 2007;179(5):3325-3331.

58. Miller YI, Tsimikas S. Oxidation-specific epitopes as targets for biotheranostic applications in humans: biomarkers, molecular imaging and therapeutics. Curr Opin Lipidol. 2013;24(5):426-437.

59. Moore TL, Gillian BE, Crespo-Pagnussat S, Feller L, Chauhan AK. Measurement and evaluation of isotypes of anti-citrullinated fibrinogen and anti-citrullinated alpha-enolase antibodies in juvenile idiopathic arthritis. Clin Exp Rheumatol. 2014;32(5):740-746

60. Gilliam BE, Reed MR, Chauhan AK, Dehlendorf AB, Moore TL. Evidence of fibrinogen as a target of citrullination in IgM rheumatoid factor-positive polyarticular juvenile idiopathic arthritis. Pediatr Rheumatol Online J. 2011;9:8.

61. Petty RE, et al. International League of Associations for Rheumatology classification of juvenile idiopathic arthritis: second revision, Edmonton, 2001. J Rheumatol. 2004;31(2):390-392.

62. Clement CC, Rotzschke O, Santambrogio L. The lymph as a pool of self-antigens. Trends Immunol. 2011;32(1):6-11.

63. Clement CC, et al. Protein expression profiles of human lymph and plasma mapped by 2D-DIGE and 1D SDS-PAGE coupled with nanoLC-ESI-MS/MS bottom-up proteomics. J Proteomics. 2013;78:172-187.

64. Robinson LZ, Reixach N. Quantification of quaternary structure stability in aggregation-prone proteins under physiological conditions: the transthyretin case. Biochemistry. 2014;53(41):6496-6510.

65. Yin L, Stern LJ. Measurement of peptide binding to MHC class II molecules by fluorescence polarization. Curr Protoc Immunol. 2014;106:5 